\title{
Inertia-gravity waves generated by near balanced flow in 2-layer shallow water turbulence on the $\beta$-plane
}

\author{
A. Wirth \\ LEGI/MEIGE/CNRS, INSU, Observatoire des Sciences de l'Univers de Grenoble (OSUG), UMS832, \\ BP 53, Grenoble, France
}

Correspondence to: A. Wirth (achim.wirth@hmg.inpg.fr)

Received: 21 June 2012 - Revised: 18 October 2012 - Accepted: 4 December 2012 - Published: 8 January 2013

\begin{abstract}
Using a fine resolution numerical model $\left(4000^{2} \times 2\right.$ grid-points) of the two-layer shallow-water equations of the mid-latitude $\beta$-plane dynamics, it is shown that there is no sudden breakdown of balance in the turbulent enstrophy cascade but a faint and continuous emission of inertia-gravity waves. The wave energy accumulates in the equator-ward region of the domain due to the Coriolis parameter depending on the latitude and dispersion relation of inertia-gravity waves.
\end{abstract}

\section{Introduction}

There now is a substantial body of evidence showing that important aspects of the large scale dynamics of the atmosphere and the ocean, away from the tropics, are very well described by the quasi-geostrophic $(\mathrm{QG})$ model. The range of its validity spans from the largest scales down to the synoptic scale (called meso scale in the ocean). The turbulent dynamics within these ranges, called QG turbulence, has been studied extensively in the last four decades (Rhines, 1975, 1979) and is now part of textbooks (Vallis, 2006). These studies have contributed substantially to our understanding of the non-linear dynamics in the atmosphere and the ocean. The driving force of the atmosphere acts at planetary scale by differential heating of the land and water surfaces by the Sun. The ocean dynamics are mostly forced by the atmospheric wind field at its surface at basin scale. The large scale circulations are unstable at the synoptic scale, which is of the order of $1000 \mathrm{~km}$ in the atmosphere and $100 \mathrm{~km}$ in the ocean at mid latitudes. The QG model has an inverse cascade of energy. The energy present at the synoptic scale is transported systematically to larger scales. An energy cascade to smaller scales is inhibited by the conservation of (potential) vorticity (Kraichnan, 1967). This picture of the QG turbulence leaves open the question of the dissipation of energy in the atmosphere and the ocean which can only happen at small scales, that is, at scales that are not systematically fed by the dynamics. Note that bottom friction seems to be insufficient to explain the loss of energy when the ocean dynamics are considered (Ferrari and Wunsch, 2009). The above leads to the conclusion that the QG model, although it is a very good one, does not tell us the whole story and it does not tell us how the energy is systematically transported to the small scales, where it can be dissipated (Bofetta and Ecke, 2012). A consequence of this is that if QG models are integrated numerically on finer and finer grids, the explicit viscosity can be decreased. In the absence of a forward cascade this leads to less and less energy being dissipated.

The energetics of the ocean (Ferrari and Wunsch, 2009) and the failure of the QG formalism have recently been gaining attention. One possible "failure" of QG is the emission of inertia-gravity waves (IGWs) in flows which are supposed to be or are very closed to a QG dynamics (Ford et al., 2000). This emission of gravity waves by the almost QG dynamics is often qualified as spontaneous, making reference to the fact that the emission is due to the internal balanced dynamics and not an initial or exterior source of imbalance. I will show in my calculation that it is, however, not spontaneous in the sense of impulsive or sudden, in agreement with other published work. Abrupt emission of IGWs has been observed, however, in the case of the frontal collapse studied by Griffiths and Reeder (1996) and Reeder and Griffiths (1996).

The analogy between emission of sound by almost incompressible (low Mach number) turbulent motion and the emission of gravity waves by almost QG motion is strong and at 
A. Wirth: Inertia-gravity waves generated by near balanced flow

the origin of the research of gravity wave emission. In their pioneering work Ford et al. (2000) adapted the formalism developed by Lighthill (1952) for the emission of sound by turbulent flow to the case of the emission of IGWs by shallow water flow on the f-plane. If one listens to subsonic noise of a turbulent flow, you do not hear any spontaneous banging sound but rather a background growl, and one expects the same thing to happen for QG flow at low Froude and Rossby number. Of course, when the flow becomes supersonic an impulsive barking noise occurs, and one expects the same thing to happen when a strict criteria of balanced dynamics is broken (see Molemaker et al., 2005 and references therein).

The absence of a direct energy cascade in 2-D turbulence emphasises the importance of turbulence. Even if it is not fed by an external source of energy, it does not fade away quickly (as is the case for 3-D turbulence) but persists for a long time, leading to a strong entangling and mixing motion. In 2-D, turbulent processes that drain energy form the vortical flow, such as gravity wave emission, maybe important although they are sub-dominant in 3-D dynamics. More precisely, even if the generation of sound in 3-D dynamics is energetically insignificant as compared to the forward energy cascade, it might well be a dominant process when the loss of energy for the 2-D vortical dynamics is considered, as there is no forward energy cascade.

There is a growing interest in the field of gravity-wave emission and there is a substantial body of evidence based on analytical calculations that IGWs are emitted from balanced flow (see, e.g. Ford et al., 2000; Vanneste and Yavneh, 2004; Lott et al., 2010 and references therein). Data from observations and experiments are scant. Numerical evidence has evolved from considering one-layer shallow-water turbulence on the f-plane (some twenty years ago, e.g. Farge and Sadourny, 1990; Polvani et al., 1994) to coherent structures, dipoles and fronts in 3-D atmospheric-models (O'Sullivan and Dunkerton, 1995; Plougonven and Snyder, 2005; Snyder et al., 2007). The value of the explicit dissipation parameter used in the calculations (viscous or hyper-viscous), which is imposed by the numerical resolution, is found to have a strong impact on the the gravity-wave dynamics in some experiments (O'Sullivan and Dunkerton, 1995). This is especially true when gravity waves trapped in vortical structures are subject to strain, which reduces their wave length (Plougonven and Snyder, 2005; Snyder et al., 2007; McIntyre, 2009).

In the present work I reconsider the shallow water turbulence, extending it to two-layer flow on the $\beta$-plane and considering a much wider range of scales in space and time than it was possible over $20 \mathrm{yr}$ ago. Nowadays fine resolution studies are done solving the hydrostatic equations with many vertical levels (see, e.g. Klein et al., 2008). The inertiagravity wave analysis is then difficult to perform as there are as many vertical modes as there are levels, all having a frequency just above inertial. Furthermore, the density structure changes in time and space, changing the dispersion relation
Table 1. Physical parameters

\begin{tabular}{ll}
\hline$h_{1}(\mathrm{~m})$ & 600 \\
$h_{2}(\mathrm{~m})$ & 1400 \\
$g\left(\mathrm{~m} \mathrm{~s}^{-2}\right)$ & 10.0 \\
$g^{\prime}\left(\mathrm{m} \mathrm{s}^{-2}\right)$ & $2.010^{-2}$ \\
$f\left(\mathrm{~s}^{-1}\right)$ & $10^{-4}$ \\
$\beta\left((\mathrm{m} \mathrm{s})^{-1}\right)$ & $10^{-11}$ \\
$L_{x}=L_{y}(\mathrm{~m})$ & $3.0 \times 10^{6}$ \\
\hline
\end{tabular}

and making long-time analysis, as done in the present work, difficult.

The physical parameters in the present calculations are typical for the turbulent mid-latitude ocean dynamics. I consider the problem of gravity-wave emission in the turbulent enstrophy cascade in calculations that span three orders of magnitude in both horizontal directions. In this way I address the question of the importance of the submesoscale dynamics in the generation of IGWs. The key question I consider is this: Does balance break down at small scales in the enstrophy cascade?

For many applications of spontaneous imbalance a continuous stratification is important. Such processes cannot be addressed by the here employed 2-layer model. For a discussion of this mechanism and a recent review of the subject of spontaneous wave generation, I refer the reader to McIntyre (2009) and Vanneste (2013) and references therein.

\section{Model}

\subsection{Physical model}

The model considered consists of two fluid layers of thickness $h_{i}$ and density $\rho_{i}$, with $i=1$ for the upper layer. The dynamics take place in a square domain with measures $3000 \mathrm{~km}$ in both horizontal directions. In the $\mathrm{x}$-direction the domain is periodic and there are free slip boundaries at $y= \pm 1500 \mathrm{~km}$. The fluid is subject to a gravitational acceleration $g$ and a rotation rate that varies linearly with latitude, $f(y)=f_{0}+\beta y$, ( $\beta$-plane).

The physical parameters given in Table 1 lead to a barotropic radius of deformation of $R_{\mathrm{bt}}=2000 \mathrm{~km}$ and a baroclinic of $R_{\mathrm{bc}}=35 \mathrm{~km}$ in the centre of the domain. The synoptic scale, that is, the size of the synoptic eddies in the ocean, is roughly a decade larger than the baroclinic radius of deformation at mid-latitudes (see Chelton et al., 2011). The ratio of the Coriolis parameter between the southern and the northern boundary is almost a factor of two. The initial condition are two dipoles of different strength that collide: 


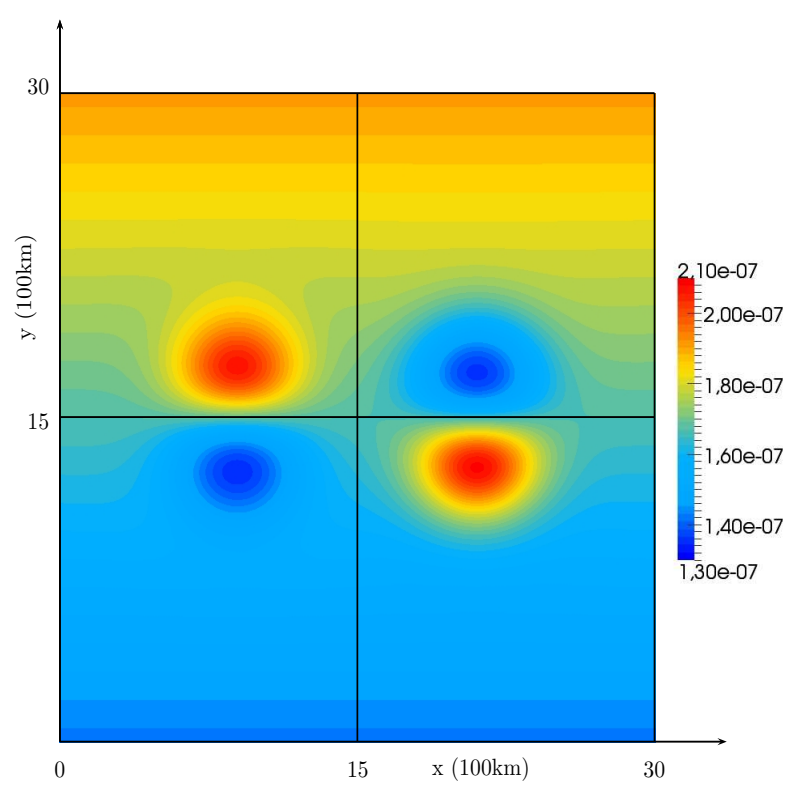

Fig. 1. Initial potential vorticity (in $\left(\mathrm{m} \mathrm{s}^{-1}\right)$ of the upper layer. The initial condition is purely large-scale. The two dipoles on the $\beta$ plane are clearly visible.

$$
\begin{aligned}
& \eta_{1}(t=0)=5 \mathrm{~m} \times\left(\exp \left[-18\left((x / l-0.4)^{2}+(y / l-0.05)^{2}\right)\right]\right. \\
& \left.-\exp \left[-18\left((x / l-0.4)^{2}+(y / l+0.05)^{2}\right)\right]\right) \\
& -4 \mathrm{~m} \times\left(\exp \left[-\left(18(x / l+0.4)^{2}+(y / l-0.05)^{2}\right)\right]\right. \\
& \left.-\exp \left[-18\left((x / l+0.4)^{2}+(y / l+0.05)^{2}\right)\right]\right) \\
& \eta_{2}(t=0)=-0.1 \times\left(g^{\prime \prime} / g^{\prime}\right) \eta_{1}(t=0)
\end{aligned}
$$

where the origin of the coordinate system is in the centre of the region and $l=1500 \mathrm{~km}$ (see Fig. 1). The initial velocity is geostrophic based on the constant Coriolis parameter $f_{0}$ :

$$
\begin{gathered}
u_{1}=-\frac{g}{f_{0}} \partial_{y} \eta_{1}, \quad v_{1}=\frac{g}{f_{0}} \partial_{x} \eta_{1}, \\
u_{2}=-\frac{g^{\prime \prime}}{f_{0}} \partial_{y} \eta_{1}-\frac{g^{\prime}}{f_{0}} \partial_{y} \eta_{2}, \quad v_{2}=\frac{g^{\prime \prime}}{f_{0}} \partial_{x} \eta_{1}+\frac{g^{\prime}}{f_{0}} \partial_{x} \eta_{2} .
\end{gathered}
$$

This initialisation procedure insures a divergence-free initial velocity field. The initial departure from geostrophy, due to a varying Coriolis parameter, leads to a wave emission by a classical (Rossby, 1938) adjustment process (Gill, 1976; Vallis, 2006), which leads to the dynamics approaching balance. Initially the barotropic Froude number is $F_{\mathrm{bt}}=8.10 \times$ $10^{-3}$ and the baroclinic Froude number is $F_{\mathrm{bc}}=3.31 \times 10^{-1}$; both numbers are calculated based on the maximal initial speed and the speed of the barotropic and baroclinic gravity waves.

The advantage of a two-layer rather than a one-layer model is twofold. The first is realism. Important aspects of ocean dynamics are well described by two layers that are separated by a sharp thermocline. Second, using a single reduced gravity layer with a realistic reduced gravity constant ( $g \approx 2.0 \times 10^{-2} \mathrm{~m} \mathrm{~s}^{-2}$ ) severely constraints the magnitude of the geostrophic transports and thus of the large scale velocities to rather feeble values. This results in a very slow evolution of the balanced dynamics and an increased stiffness, which is a large separation in the characteristic time scales between vortical dynamics and wave dynamics. In the twolayer model, the stiffness can be reduced (i) as the barotropic mode supports high velocities, which lead to a strong vortical dynamics in both layers; and (ii) as the baroclinic mode is advected by the barotropic mode. Please note that the initial condition (Eqs. 1 and 2) has a dominant barotropic mode. Due to the large difference between the baroclinic and the barotropic Froude number, baroclinc waves have a much larger amplitude than the barotropic counterpart. In the analysis of the baroclinc waves, I can thus restrict myself to the upper-layer dynamics.

\subsection{Mathematical model}

The mathematical model used to study the physical mode, applies the classical two-dimensional two-layer shallow water (SW) equations on the $\beta$-plane. The SW equations not only represent the advection of vorticity but also include vortex stretching and the dynamics of IGWs. Their dynamics are thus more involved than the 2-D Navier-Stokes equations, which only contain the advection of vorticity. They are also more involved than QG dynamics, which contain the advection of vorticity and vortex stretching but no IGWs. The densities in the layers $i=1,2$ are $\rho_{i}$ and the average thicknesses are $h_{i}^{0}$. The horizontal components of the vertically-averaged velocity vector are $u_{i}(t, x, y)$ (longitudinal direction) and $v_{i}(t, x, y)$ (latitudinal direction). The deviation from a flat surface and interface is denoted by $\eta_{1}(t, x, y)$ and $\eta_{2}(t, x, y)$, respectively. Note that the total thickness of the upper layer is $\left(h_{1}^{0}+\eta_{1}-\eta_{2}\right)$. The governing equations are

$$
\begin{aligned}
& \partial_{t} u_{1}+\quad u_{1} \partial_{x} u_{1}+v_{1} \partial_{y} u_{1}+g \partial_{x} \eta_{1}-f v_{1}=v \nabla^{2} u_{1} \\
& \partial_{t} v_{1}+\quad u_{1} \partial_{x} v_{1}+v_{1} \partial_{y} v_{1}+g \partial_{y} \eta_{1}+f u_{1}=v \nabla^{2} v_{1} \\
& \left.\partial_{t} \eta_{1}+\partial_{x}\left[\left(h_{1}^{0}+\eta_{1}-\eta_{2}\right) u_{1}\right]+\partial_{y}\left[\left(h_{1}^{0}+\eta_{1}-\eta_{2}\right) v_{1}\right)\right]+ \\
& \left.\partial_{x}\left[\left(h_{2}^{0}+\eta_{2}\right) u_{2}\right]+\partial_{y}\left[\left(h_{2}^{0}+\eta_{2}\right) v_{2}\right)\right]=\kappa \nabla^{2} \eta_{1} \\
& \partial_{t} u_{2}+u_{2} \partial_{x} u_{2}+v_{2} \partial_{y} u_{2}+g^{\prime \prime} \partial_{x} \eta_{1}+g^{\prime} \partial_{x} \eta_{2}-f v_{2} \\
& =v \nabla^{2} u_{2} \\
& \partial_{t} v_{2}+\quad u_{2} \partial_{x} v_{2}+v_{2} \partial_{y} v_{2}+g^{\prime \prime} \partial_{y} \eta_{1}+g^{\prime} \partial_{y} \eta_{2}+f u_{2} \\
& =v \nabla^{2} v_{2} \\
& \left.\partial_{t} \eta_{2}+\quad \partial_{x}\left[\left(h_{2}^{0}+\eta_{2}\right) u_{2}\right]+\partial_{y}\left[\left(h_{2}^{0}+\eta_{2}\right) v_{2}\right)\right] \\
& =\kappa \nabla^{2} \eta_{2} \text {, }
\end{aligned}
$$

where $g$ is gravity $g^{\prime \prime}=g \rho_{1} / \rho_{2}$ and $g^{\prime}=g\left(\rho_{2}-\rho_{1}\right) / \rho_{2}=$ $g-g^{\prime \prime}$. The boundary conditions at the northern and southern boundaries are free-slip $\left(u=\partial_{y} v=0\right)$ for the velocity 
and there are no diffusive fluxes of layer thickness across the boundary. The domain is periodic in the east-west direction.

The justification of the implementation of diffusion of layer thickness $\kappa$ is given in Appendix A. This GentMcWilliams parameter is used in the same spirit as the viscosity parameter. Their role is to prevent the accumulation of energy/enstrophy at the smallest scales that are resolved numerically. Please see Frisch et al. (2008) for a detailed discussion of this bottleneck phenomena. The explicit values of the thickness-diffusion and the viscosity parameter depend on the resolution of the numerical model.

Two important scalar fields for each layer that can be formed are the potential vorticity (PV) and the divergence. The potential vorticity in the upper layer is

$q_{1}=\frac{\partial_{x} v_{1}-\partial_{y} u_{1}+f}{h_{1}^{0}+\eta_{1}-\eta_{2}}$,

which is the total vorticity, relative vorticity plus planetary vorticity, divided by the layer thickness. If viscosity and diffusion of layer thickness vanishes the potential vorticity is advected by the flow:

$\left(\partial_{t}+u_{1} \partial_{x}+v_{1} \partial_{y}\right) q_{1}=0$.

The potential vorticity of inertial-gravity waves vanishes to leading order, so that they cannot be detected in the PVfield. As the advecting velocity field is not divergence free the space average of the PV is however varying in time. As the divergence is small so is the variation of PV. The divergence,

$d_{1}=\partial_{x} u_{1}+\partial_{y} v_{1}$

vanishes for geostrophic flow and is small for QG-motion but clearly emphasises inertial-gravity waves.

Using Eqs. (3)-(8), a wave equation for the divergence in the upper layer can be derived. The details of this derivation are given in Appendix A. Equation (A2) shows the classical inertia-gravity wave equation (see e.g. Vallis, 2006) with a term $r_{1}$ on the right, which is detailed in Eq. (A3). The righthand side $r_{1}$ is referred to as the source term, but not all its terms are necessarily a source of gravity waves, as discussed in Appendix A.

\subsection{Numerical model}

The numerical model chosen to solve the above detailed mathematical model is a second-order-in-time Runge-Kutta scheme. The spatial discretisation is a centred second order in space scheme (first order at the boundary). The finest resolution has $4000^{2}$ points $(750 \mathrm{~m}, \Delta t=1.5 \mathrm{~s})$, three calculations were also performed with $2000^{2}$ points $(1.5 \mathrm{~km} \Delta t=3 \mathrm{~s})$. The short time-step is imposed by the barotropic gravity wave speed. For details please see Table 2.

The wave dynamics is shown to be very sensitive to the numerical scheme and the time-step and spacial resolution used (Farge and Sadourny, 1990; Polvani et al., 1994). I performed
Table 2. Parameters varied between numerical experiments.

\begin{tabular}{lrrr}
\hline $\exp$ & $n_{x}=n_{y}$ & $\nu=\kappa\left(\mathrm{m}^{2} \mathrm{~s}^{-1}\right)$ & length (days) \\
\hline exp11 & 4000 & 1 & 275 \\
$\exp 21$ & 2000 & 1 & 250 \\
$\exp 22$ & 2000 & 10 & 500 \\
$\exp 23$ & 2000 & 100 & 250 \\
\hline
\end{tabular}

simulations with different time-step and spatial resolution. Note that my calculations are performed at a spacial resolution that has 400 times more grid points than the pioneering work (Farge and Sadourny, 1990; Polvani et al., 1994) done $20 \mathrm{yr}$ ago. Please also note that during the longest experiment performed (exp22, see Table 2$)$ a short baroclinic gravity wave has travelled about $1.5 \times 10^{8} \mathrm{~m}$, which is 50 times the domain size; a bartopic wave is about 40-times faster. The dynamics of barotropic gravity waves crossing half of the domain in between two consecutive snapshots (every $3 \mathrm{~h}$ ) cannot be studied. Their signal is, however, small as the barotropic Froude number is tiny (see Sect. 2.1).

The results presented in Sect. 3 clearly indicate a good convergence of the numerics employed.

The dissipation constants, viscosity and layer-thickness diffusion adapted for the resolution employed are $v=\kappa=$ $10^{\alpha} \mathrm{m}^{2} \mathrm{~s}^{-1}$ with $\alpha=0,1$ and 2 . The experiment with the coarser resolution and the lowest friction value (exp21) was under-resolved towards the end of the integration. A calculation with $\alpha=3$ (not discussed) leads to a fast decay of vortical and IGW motion. For the viscosities with $\alpha=2$ and 3, calculations were also performed with a resolution of $500 \times 500$ points. The evolution of the PV field showed no substantial differences to the calculations with higher resolution presented here, but the wave dynamics were substantially different. This again points to the high sensitivity of the wave dynamics to the numerics employed. I have learned in the present research that a single calculation on wave dynamics might not give reliable outcome; a series of experiments with varying spatial and temporal resolution is necessary to verify the convergence of the results.

Using even higher resolutions might not add much to our understanding of the physics involved, as the approximations (e.g. hydrostatic-approximation) at the basis of the shallow water model are no longer valid for dynamical phenomena that have horizontal extension comparable to the water depth $(2 \mathrm{~km})$. Even higher resolution might teach us things about the mathematics of the SW model but not about the physics of the ocean and/or atmosphere. 


\section{Results}

\subsection{Qualitative description}

In this work I focus on the duality between vortical and wave dynamics and their interaction. The vortical dynamics are described by the PV and the wave dynamics are explored through the divergence.

Initially there are two dipoles facing each other as shown in Fig. 1. As the collision of the dipoles happens on the $\beta$ plane, the conservation of PV prevents the vortical dynamics to penetrate far to the north or the south, as can be verified in Fig. 2. Non-linear interaction leads to a filamentation and erosion of the coherent vortices. Smaller and smaller vortical structures arise, which is a manifestation of the enstrophy cascade towards small scales. Several sharp fronts are created. Submesoscale vortices appear on these fronts and can be identified in Fig. 2.

The initial condition is close to but not completely in a balanced state. This leads initially to a classical geostrophic adjustment (Rossby, 1938; Gill, 1976) with emission of gravity waves. The barotropic gravity waves decay rapidly in all experiments and subsequently stay below the noise level in all the experiments. The vortical structures continue to emit gravity waves during the entire experiment. The major part of the vortical dynamics rests confined to the mid-latitudes of the domain due to the conservation of potential vorticity and the varying Coriolis parameter. The IGWs accumulate in the lower latitudes of the domain. This spatial separation helps in dividing vortical and IGW dynamics.

Contrary to what was observed by Polvani et al. (1994), no spontaneous generation of bores and shocks are seen in the experiments.

\subsection{Potential vorticity (PV) dynamics}

The potential vorticity anomaly (PVA),

$a_{1}=q_{1}-\frac{f}{h_{1}^{0}}$,

gives the potential vorticity minus the potential vorticity of a motionless ocean. It is a perfect indicator for the vortical dynamics. The vortical dynamics do not penetrate far to the south or north, see Fig. 2, but stay in the mid-latitudes of the domain due to the conservation of $\mathrm{PV}$ on the $\beta$-plane. As a measure of the total vortical dynamics, I choose

$\overline{a_{1}}=\sqrt{\left\langle q_{1}^{2}\right\rangle-\left\langle\left(\frac{f}{h_{1}^{0}}\right)^{2}\right\rangle}$,

where $\langle$.$\rangle denotes the horizontal-space average over the$ whole domain. Note that $\overline{a_{1}}$ is not a conserved quantity (see Sect. 2.2); even when viscosity and layer diffusivity vanish, $v=\kappa=0$. The enstrophy cascade to small scales, which acts by stretching of the PV structures, can be seen by comparing Figs. 1 and 2. At the small scales the PV is then dissipated by

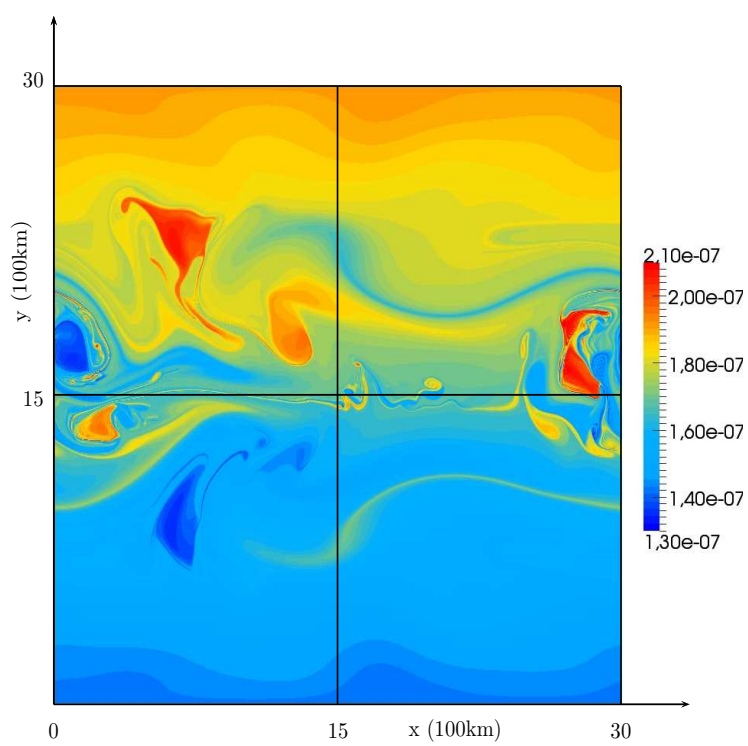

Fig. 2. Potential vorticity (in $(\mathrm{m} \mathrm{s})^{-1}$ ) after 250 days in exp11. The enstrophy cascade has transported potential vorticity to small scales. The two dipoles have disintegrated, leaving small scale vortices, filaments and fronts behind. They are a signature of the turbulent enstrophy cascade.

the direct action of viscosity and dissipation of layer thickness, leading to an overall decline of $\overline{a_{1}}$. After 250 days the decrease of $\bar{a}_{1}$ is only weakly dependent on viscosity as it is governed by the flux of PV from large to small scales. So to first order, an increased viscosity leads to an earlier dissipation of PV but not a stronger one. The enstrophy cascade leads to a finite dissipation of enstrophy in the limit of vanishing dissipation (Frisch, 1995). The $\overline{a_{1}}$ has decreased by 12-22\% (depending on the exp., see Fig. 4) during the first 250 days. Energy, a quantity that cascades to large scales, has decreased by only $0.14 \%$ in exp11 and exp 21 , by $1.2 \%$ in exp22, and slightly less than $10 \%$ in exp23 during the same period, reflecting the variation of the dissipation parameters. Note also that the major part of energy at large scales (larger than Rossby radius) is stored in form of available potential energy rather than kinetic energy.

The time-evolution of the length scale,

$\lambda_{\zeta}=2 \pi \sqrt{\frac{\left\langle\zeta_{1}^{2}\right\rangle_{m}}{\left\langle\left(\nabla \zeta_{1}\right)^{2}\right\rangle_{m}}}$

is shown in Fig. 6. It is two-pi times the r.m.s. (root mean square) enstrophy divided by the r.m.s. palinstrophy (see Frisch, 1995). It is characteristic of viscous dissipation length scales in the enstrophy cascade (Bofetta and Ecke, 2012), the smallest scales in the vortical dynamics. The spatial averages \langle\rangle$_{m}$ are taken over latitudinal middle-quarter of the domain. This is roughly the area to which the vortical dynamics are constraint by the conservation of potential vorticity on the $\beta$-plane (see Fig. 2). The length scale $\lambda_{\zeta}$ decreases rapidly 


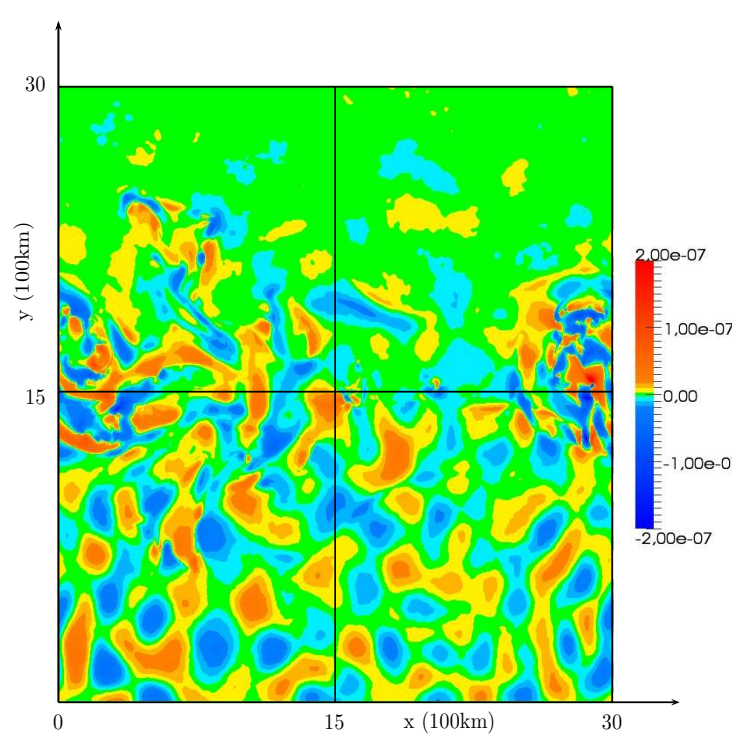

Fig. 3. Divergence of the upper layer (in $s^{-1}$ ) after 250 days in exp11. The accumulation of wave activity is clearly visible in the southern part of the domain. As is the dominance of structures of scale above the (baroclinic) deformation radius.

during the first 70 days in all experiments, indicating the spreading of the enstrophy cascade towards smaller scales. The decrease is almost independent of viscosity for the first 50 days and is then stopped by viscosity. At these scales, enstrophy is dissipated by viscosity before it can reach even smaller scales. For the lowest viscosity value the size is just above $12 \mathrm{~km}$ (which is 16 times the grid size). The strong decrease of more than one decade is a clear signature of the enstrophy cascade. It also shows the non-stationarity of the vorticity dynamics in this decaying turbulence experiment.

\subsection{Inertia-gravity wave (IGW) dynamics}

The divergence can be decomposed into two parts, (i) the divergence of the vortical and (ii) the wave dynamics $d_{1}=$ $d_{1}^{\mathrm{QG}}+d_{1}^{\mathrm{W}}$. They can be separated by considering the time variability, as the frequency of the wave field is bounded from below by the Coriolis parameter $f$. The vortical part is calculated as a box average over $30 \mathrm{~h}$ from the actual divergence field

$d_{1}^{\mathrm{QG}}(t)=\frac{1}{30 \mathrm{~h}} \int_{-15 \mathrm{~h}}^{15 \mathrm{~h}} d_{1}\left(t+t^{\prime}\right) \mathrm{d} t^{\prime}$.

The average over results concerning the divergence have an oscillatory component, as shown in Fig. 5. The reason for this is mostly the aliasing of high-frequency baroclinic and barotropic waves; such high frequency waves cannot be resolved by 3-hourly snapshots. Pollution by barotropic gravity waves is difficult to avoid; they have phase and group velocities exceeding $140 \mathrm{~m} \mathrm{~s}^{-1}$, crossing the entire domain in about

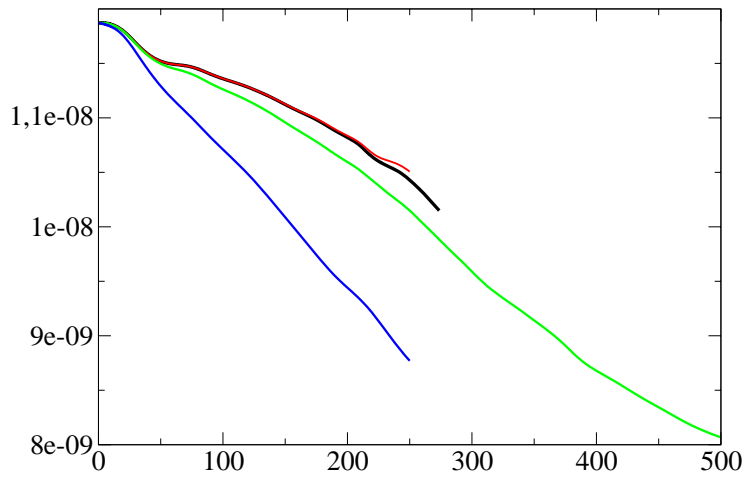

Fig. 4. Quantity $\overline{a_{1}}$ (see Eq. 9 in the text) (in $(\mathrm{m} \mathrm{s})^{-1}$ ) measuring the vortical dynamics in the different experiments, as a function of time (in days), for experiments 01 (black) 11 (red), 12 (green) and 13 (blue). Due to the enstrophy cascade there is a fast decrease in vortical dynamics, which depends only weakly on the value of the viscosity parameter. The black and the red curve presenting the same mathematical model (same viscosity) at different resolution, separate after approx 220 days, indicating that the lower resolution run (red) is (slightly) under-resolved.

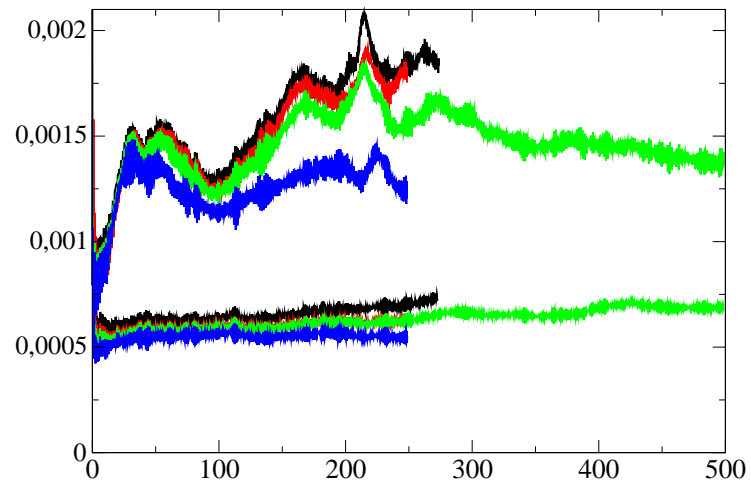

Fig. 5. Upper curves: r.m.s. of the divergence of the upper layer averaged the whole domain divided by the r.m.s of the potential vorticity anomaly of the upper layer, and the average layer thickness, averaged the whole domain, as a function of time (in days) for exp11 (black) exp21 (red), exp22 (green) and exp23 (blue). Lower curves: same as upper, except that the 30-h time average of the divergence is subtracted to obtain the wave part of the divergence.

$6 \mathrm{~h}$ and cannot be filtered out with snapshots every $3 \mathrm{~h}$. Their amplitude is however small, so that they are only barely visible even when the divergence is considered.

Such oscillations are almost perfectly absent in the potential vorticity dynamics (see Fig. 4) as its signal is about 1000 times stronger and waves are mostly filtered out. Figure 3 shows an absence of wave energy in the northern half of the domain, which can be explained by the dispersion relation of IGWs:

$\omega^{2}=c^{2}\left(k_{x}^{2}+k_{y}^{2}\right)+f^{2}$, 


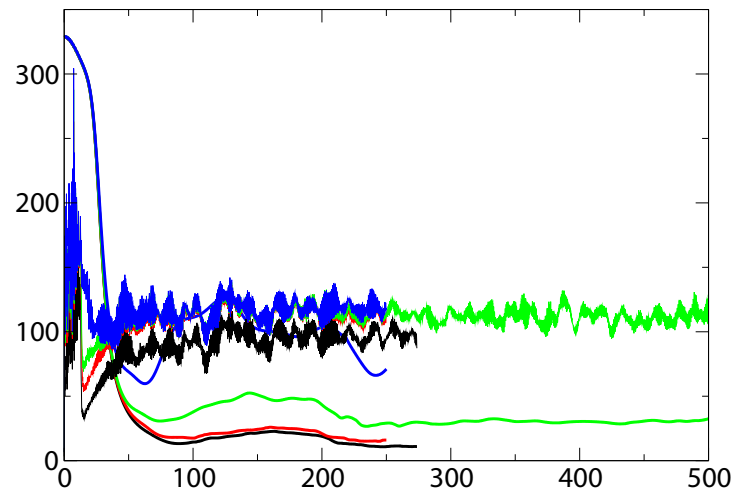

Fig. 6. Smooth curves: characteristic horizontal size $\lambda_{\zeta}$ (in $\mathrm{km}$ ) of the vorticity field in the latitudinal middle quarter of the domain, in the upper layer. Wiggly curves: characteristic horizontal size $\lambda_{d}$ (in $\mathrm{km}$ ) of the divergence field in southern quarter of the domain, in the upper layer. Both are given as a function of time (in days), for exp11 (black), exp21 (red), exp22 (green) and exp23 (blue).

with $f=f_{0}+\beta y$. When waves propagate northward the magnitude of their latitudinal wave number has to decrease. When it can no longer decrease, $k_{y}^{2}=0$, the critical latitude is reached (see Pedlosky, 2003, p. 193). If the generation happens around the local inertial frequency, the lowest allowed for inertia-gravity waves, then the energy can only proceed equator-ward. This behaviour was called $\beta$-dispersion in Anderson and Gill (1979) and explored for the one dimensional case using ray-theory. The extension to two dimensions is given in Appendix B. The transport of inertia-gravity wave energy towards low latitudes was also observed in ocean data by Alford (2003). Another important point is the increase of divergence of the inertia-gravity wave when moving equatorward (see Appendix B for further details). The spatial distribution of divergence seen in Fig. 5 is therefore explained by the $\beta$-dispersion and an increase of divergence IGWs moving towards lower latitudes. To further determine the behaviour of inertia-gravity waves on the $\beta$-plane, a wave-turbulence theory is required, which does not exist so far.

The slowly varying part of the divergence $d^{\mathrm{QG}}$ is vanishing in the northern and southern part of the domain.

To compare the vortical dynamics to the divergent dynamics, I consider the non-dimensional quantities:

$\bar{d}_{1}=\frac{\sqrt{\left\langle d_{1}^{2}\right\rangle}}{\overline{a_{1} h_{1}^{0}}} \quad$ and $\quad \overline{d_{1}^{\mathrm{W}}}=\frac{\sqrt{\left\langle\left(d_{1}^{\mathrm{W}}\right)^{2}\right\rangle}}{\overline{a_{1} h_{1}^{0}}}$,

which normalises the divergent (wave) dynamics by the vortical dynamics. The results are presented in Fig. 5. The normalised divergence $\left(\bar{d}_{1}\right)$ shows a slow time variability with a maximum occurring at around day 210 . The normalised wave divergence $\left(d_{1}^{\overline{\mathrm{W}}}\right)$ is, after a rapid initial decrease, almost constant at an amplitude above $5.0 \times 10^{-4}$ with a slight increase in time. This level is small but clearly non-vanishing. The space-averaged divergence of the wave part (without being divided by $\bar{a}_{1}$ ) grows in time for the highest resolution experiment, exp11. The amplitude of the wave part does not vary much between experiments, which points to the fact that it is not very sensitive to the magnitude of the dissipation, which is varied by two orders of magnitude between experiments. Furthermore, exp21 is clearly under-resolved towards the end of the experiment, which can be seen in Fig. 4 where the graph starts to diverge from the finer resolution calculation of exp11, indicating an accumulation of vorticity at the smallest scale that cannot be dissipated sufficiently fast - a phenomenon referred to as "bottelneck" (see Frisch et al., 2008 for a detailed discussion). That this under-resolution does not lead to an increase in wave activity is an important point, as most operational models in oceanography or meteorology are under-resolved and as higher-order dissipation-operators (hyper-viscosity), widely used in the numerical modelling of the ocean and the atmosphere dynamics, also lead to a "bottleneck". Furthermore, the insensitivity of the wave amplitude to the value of the dissipation parameters demonstrates that dissipation is not a source of gravity waves, which could be estimated by a naive inspection of Eq. (A3), as the terms involving viscosity clearly appear as a source term in the gravity wave equation derived using the Lighthill/Ford formalism.

The time evolution of the characteristic size of the divergence field is investigated by considering the length scale:

$\lambda_{\mathrm{d}}=2 \pi \sqrt{\frac{\left\langle d_{1}^{2}\right\rangle_{\mathrm{s}}}{\left\langle\left(\nabla d_{1}\right)^{2}\right\rangle_{\mathrm{s}}}}$,

which is the equivalent of divergence to the length scale $\lambda_{\zeta}$ for vorticity. The spatial averages \langle\rangle$_{\mathrm{s}}$ are taken over the southern quarter of the domain. This is roughly the area where the most wave activity is present and the vortical dynamics are absent (see Figs. 2 and 3). In the centre of the domain, the divergence is dominated by the divergence due to the vortical dynamics, as can be seen in Fig. 5. The vortical dynamics can furthermore lead to wave capture and distortion of the gravity wave field, leading to small size structures and change of the background vorticity in space and to Doppler-shifts in the frequency. For details see Appendix A and Kunze (1985), Zhai et al. (2005), McIntyre (2009). The typical size of the divergence in the southern quarter of the domain settles at around $100 \mathrm{~km}$ in all experiments, with only a weak dependence on the viscosity. This shows that in the experiments presented here, the amplitude and scale of gravity-wave dynamics are almost independent of the explicit value of the dissipation parameters and the characteristic size of the vorticity field. They are also independent of the characteristic scale of the vorticity field that has decreased by over one order of magnitude. This agrees with the results of Ford et al. (2002) (see also McIntyre, 2009), who found that the emitted wave frequency is very close to the inertial frequency, leading to large scale inertia-gravity 
waves, as seen from the dispersion relation (11). As the wave packet moves towards lower latitudes, the size of latitudinal wavenumber increases, as described by the $\beta$-dispersion (see Appendix B). For a more quantitative analysis a waveturbulence theory is required, which, so far, does not exist for the $\beta$-plane.

\section{Discussion}

The most interesting feature of this research is what has NOT been observed in the experiments: there are no sudden changes in the amplitude of the wave part of the divergence, as can be verified in Fig. 5. This clearly shows that there is no sudden breakdown of balance during the integration but a faint and continuous emission of gravity waves.

After a fast initial decrease of the waves emitted by the classical adjustment to balance, the wave activity normalised by the vortical activity stays almost constant or changes slowly at an almost constant rate. At the same time, vorticity cascades to smaller scales and numerous strong fronts and small eddies are observed. This clearly indicates that the enstrophy cascade does not lead to a breakdown of balance at small scales, but on the contrary the dynamics manage to keep balance even when the size of the structures falls way below the synoptic scale. This can be explained by a constant eddy-turnover time (to leading order) in the enstrophy cascade (see e.g. Vallis, 2006). The motion on the smallest scales contains low energy and does not have an increased frequency. The wave emission depends on the high frequency tail $(\geq f)$ of the gravity wave source; as emphasised by Ford et al. (2002). The high wave number part of the enstrophy cascade is not important. For the energy cascade the eddy turnover time decreases with decreasing scale, which shows that the numerical resolution of the high wave number range of the energy cascade is important for gravity wave generation. I have not observed spontaneously-emitted bores of gravity wave fronts, although sharp fronts in the potential vorticity are numerous towards the end of the experiment.

I have to mention that while the shallow water model manages to produce sharp fronts and small-scale structures, the frontal instabilities are usually 3-dimensional and nonhydrostatic and are excluded in the SW model. In nonhydrostatic models of the same physical problem, small-scale non-hydrostatic instability are likely to occur. Such kind of behaviour was clearly observed in fine-resolution 3-D hydrostatic simulations of up-welled cold filaments in the California current system by Capet et al. (2008).

In the present calculations the boundary conditions are periodic and no-slip. In the real ocean the gravity waves will quickly be dissipated in the shallow regions near the boundaries. IGWs represent an efficient mechanism to drain energy from the interior, where most of the available potential and kinetic energy resides, and transport it to the boundaries where it is dissipated. In the present example the wave motion is small when compared to the vortical motion, but it is found (ongoing research) to strongly increase when topography is included. Again, there is a strong analogy to the generation of sound by subsonic flow, which we can mostly hear when it interacts with solid structures.

\section{Appendix A}

\section{Lighthill/ford formalism}

When the governing equations are written in a form where then non-linear and dissipative terms are on the right, we have

$$
\begin{aligned}
\partial_{t} u_{1} & +g \partial_{x} \eta_{1}-f v_{1}=-u_{1} \partial_{x} u_{1}-v_{1} \partial_{y} u_{1}+v \nabla^{2} u_{1} \\
\partial_{t} v_{1} & +g \partial_{y} \eta_{1}+f u_{1}=-u_{1} \partial_{x} v_{1}-v_{1} \partial_{y} v_{1}+v \nabla^{2} v_{1} \\
\partial_{t} \eta_{1} & +h_{1}^{0}\left(\partial_{x} u_{1}+\partial_{y} v_{1}\right)+h_{2}^{0}\left(\partial_{x} u_{2}+\partial_{y} v_{2}\right)= \\
& -\partial_{x}\left(\left(\eta_{1}-\eta_{2}\right) u_{1}\right)-\partial_{y}\left(\left(\eta_{1}-\eta_{2}\right) v_{1}\right) \\
& -\partial_{x}\left(\eta_{2} u_{2}\right)-\partial_{y}\left(\eta_{2} v_{2}\right)+\kappa \nabla^{2} \eta_{1} \\
\partial_{t} u_{2} & +g^{\prime \prime} \partial_{x} \eta_{1}+g^{\prime} \partial_{x} \eta_{2}-f v_{2}=-u_{2} \partial_{x} u_{2}-v_{2} \partial_{y} u_{2} \\
\partial_{t} v_{2} & +g^{\prime \prime} \partial_{y} \eta_{1}+g^{\prime} \partial_{y} \eta_{2}+f u_{2}=-u_{2} \partial_{x} v_{2}-v_{2} \partial_{y} v_{2} \\
\partial_{t} \eta_{2} & +h_{2}^{0}\left(\partial_{x} u_{2}+\partial_{y} v_{2}\right)=-\partial_{x}\left(\eta_{2} u_{2}\right)-\partial_{y}\left(\eta_{2} v_{2}\right)+\kappa \nabla^{2} \eta_{2} .
\end{aligned}
$$

Divergence and vorticity are

$d_{i}=\partial_{x} u_{i}+\partial_{y} v_{i}$

$\zeta_{i}=\partial_{x} v_{i}-\partial_{y} u_{i}$

and we have:

$$
\begin{aligned}
\partial_{t} d_{1}+ & g \nabla^{2} \eta_{1}-f \zeta_{1}=-\beta u_{1}+v \nabla^{2} d_{1} \\
- & \partial_{x}\left(u_{1} \partial_{x} u_{1}+v_{1} \partial_{y} u_{1}\right)-\partial_{y}\left(u_{1} \partial_{x} v_{1}+v_{1} \partial_{y} v_{1}\right) \\
\partial_{t} d_{2}+ & g^{\prime \prime} \nabla^{2} \eta_{1}+g^{\prime} \nabla^{2} \eta_{2}-f \zeta_{2}=-\beta u_{2}+v \nabla^{2} d_{2} \\
- & \partial_{x}\left(u_{2} \partial_{x} u_{2}+v_{2} \partial_{y} u_{2}\right)-\partial_{y}\left(u_{2} \partial_{x} v_{2}+v_{2} \partial_{y} v_{2}\right) \\
\partial_{t} \zeta_{1}= & -f d_{1}-\beta v_{1}+v \nabla^{2} \zeta_{1} \\
& -\partial_{x}\left(u_{1} \partial_{x} v_{1}+v_{1} \partial_{y} v_{1}\right)+\partial_{y}\left(u_{1} \partial_{x} u_{1}+v_{1} \partial_{y} u_{1}\right) \\
\partial_{t} \zeta_{2}= & -f d_{2}-\beta v_{2}+v \nabla^{2} \zeta_{2} \\
& -\partial_{x}\left(u_{2} \partial_{x} v_{2}+v_{2} \partial_{y} v_{2}\right)+\partial_{y}\left(u_{2} \partial_{x} u_{2}+v_{2} \partial_{y} u_{2}\right) .
\end{aligned}
$$

The formal equation for the divergence in both layers is

$\partial_{t t} \boldsymbol{d}+\boldsymbol{W} \boldsymbol{d}=\boldsymbol{r}$

with

$$
\begin{array}{r}
\boldsymbol{W}=\left(\begin{array}{cc}
f^{2}-g h_{1}^{0} \nabla^{2} & -g h_{2}^{0} \nabla^{2} \\
-g^{\prime \prime} h_{1}^{0} \nabla^{2} & f^{2}-g h_{2}^{0} \nabla^{2}
\end{array}\right), \\
\boldsymbol{d}=\left(\begin{array}{l}
d_{1} \\
d_{2}
\end{array}\right), \quad \boldsymbol{r}=\left(\begin{array}{l}
r_{1} \\
r_{2}
\end{array}\right) .
\end{array}
$$

The 1.h.s. of Eq. (A1) is the IGW operator. The first line of this equation reads

$$
\partial_{t t} d_{1}-g h_{1}^{0} \nabla^{2} d_{1}-g h_{2}^{0} \nabla^{2} d_{2}+f^{2} d_{1}=r_{1} ;
$$


and if we further define the Jacobian determinant and the advection operator,

$$
\begin{aligned}
D_{i} & =\left(\partial_{x} u_{i}\right)\left(\partial_{y} v_{i}\right)-\left(\partial_{x} v_{i}\right)\left(\partial_{y} u_{i}\right) \\
A_{i} . & =u_{i} \partial_{x} \cdot+v_{i} \partial_{y} .
\end{aligned}
$$

We get with some reordering

$$
\begin{aligned}
r_{1} & =-\partial_{t}\left(\beta u_{1}+A_{1} d_{1}+d_{1}^{2}-2 D_{1}\right) \\
& -f\left(\beta v_{1}+A_{1} \zeta_{1}+d_{1} \zeta_{1}\right) \\
& +g \nabla^{2}\left(\left(A_{1}+d_{1}\right) \eta_{1}+\left(A_{2}-A_{1}+d_{2}-d_{1}\right) \eta_{2}\right) \\
& +v \partial_{t} \nabla^{2} d_{1}+v f \nabla^{2} \zeta_{1}-\kappa g \nabla^{4} \eta_{1} .
\end{aligned}
$$

A non-vanishing $r_{1}$ does not necessarily mean a source of gravity waves. For example, if there is a constant large-scale velocity, the term $A_{1} d_{1}$ insures Galilean invariance, leading to a Doppler-shift that disappears when calculations are done in a co-moving frame. If the large-scale dynamic describes a solid body rotation the term $-f \zeta_{1} d_{1}$ reflects the change in total vorticity. It disappears from $r_{1}$ if equations are placed in a co-rotating frame, with a modified Coriolis parameter. More generally, variations in the vortical dynamics at scales larger than the wave length modify, to leading order, the dynamics as described by WKB-theory (see Kunze 1985 and Zhai et al., 2005).

The dissipation terms containing $\kappa$ and $v$ mimic the effect of the unresolved small scales; on the resolved dynamics their values are chosen to insure numerical stability and exceed the molecular values by many orders of magnitude. In the calculations presented here I choose $\kappa=v$, as in this case the two last dissipation terms in Eq. (A3) vanish for a flow in geostrophic equilibrium. The layer-thickness diffusivity $\kappa$ is used here, not to parameterise the effect of unresolved processes, as in the Gent-McWilliams (1990) parameterisation, but to reduce the magnitude of a source term in the wave equation due to explicit viscosity and dissipation. The $\beta$ terms are the only ones that are not invariant by rotation, as the change of the Coriolis parameter with latitude introduces rotational invariance.

To quantify and localise in detail the generation of IGWs, a Green's function integral has to be solved over the whole domain and time of integration; this is numerically not feasible. As the divergence squared is much smaller in magnitude than $D$, Eq. (A3) tells us that time variation of $D$ is a possible source of wave generation. Note also that the term $d_{i}^{2}-4 D_{i}$ is the Okubo-Weiss parameter.

The slow manifold exists only if $r_{i}=0$ on the slow manifold.

\section{Appendix B}

\section{$\beta$-dispersion}

The $\beta$ - dispersion of inertia-gravity waves explained by Anderson and Gill (1979) is based on Ray-theory (see Lighthill,
Chap. 4.5, and also Reeder and Griffiths, 1996 for an application to IGW-rays in the vertical); the starting point is that a dynamical variable can be written as

$q(x, y, t)=Q(x, y, t) \exp \left[i\left(k_{x} x+k_{y} y-\omega t\right)\right]$

with $Q(x, y, t)$ varying on spatial scales much larger than $k_{x}^{-1}$ and $k_{y}^{-1}$. The dispersion relation is independent of space and time and a wave parcel propagates with the group velocities, which depend on space:

$\partial_{t} x=C_{g}^{x}=-\frac{\partial \omega}{\partial k_{x}} \quad$ and $\quad \partial_{t} y=C_{g}^{y}=-\frac{\partial \omega}{\partial k_{y}}$.

Furthermore, we have

$\partial_{t} k_{x}=\partial_{x} \omega$ and $\partial_{t} k_{y}=\partial_{y} \omega$,

showing that $k_{x}=$ const. along a ray. Equations (B2) and (B3) show that $\omega$ plays the role of a Hamiltonian. For the $\beta$-plane $(f=\beta y)$ we get

$C_{g}^{x}=\frac{c^{2} k_{x}}{\omega}$

$C_{g}^{y}=\frac{c^{2} k_{y}}{\omega}=\frac{c \sqrt{\omega^{2}-c^{2} k_{x}^{2}}}{\omega} \sqrt{1-\frac{\beta^{2}}{\omega^{2}-c^{2} k_{x}^{2}} y^{2}}$,

which has solutions of the form

$x=\frac{c^{2} k_{x}}{\omega} t$

$y=\frac{\sqrt{\omega^{2}-c^{2} k_{x}^{2}}}{\beta} \sin \left(\frac{\beta c}{\omega} t+\phi_{t}\right)$,

where $\phi_{t}$ is the phase. Rays are of the form

$y=\frac{\sqrt{\omega^{2}-c^{2} k_{x}^{2}}}{\beta} \sin \left(\frac{\beta}{c k_{x}} x+\phi_{t}\right)$,

and the critical latitude is given by the amplitude of the sine function.

The divergence of an inertia-gravity wave is proportional to the wave number $k=\sqrt{k_{x}^{2}+k_{y}^{2}}$. When a wave packet is at its critical latitude $Y_{\text {crit }}$, then $k_{y}=0$. When it moves equatorward its energy, frequency $\omega$ and zonal wavenumber $k_{x}$ are conserved and the meridional wavenumber

$k^{2}=\frac{\omega^{2}-\beta^{2} y^{2}}{c}$

increases. The divergence

$d \propto \sqrt{\frac{\omega^{2}-\beta^{2} y^{2}}{c}}$

increases when the wave packet moves towards lower latitudes.

The $\beta$-dispersion not only depends on the planetary potential vorticity gradient but on the total PV gradient, as discussed by Kunze (1985), and that uniform currents can cause a Doppler shift of the inertial frequency, possibly leading to oscillations beyond the critical latitude (Zhai et al., 2005). 
Acknowledgements. I thank L. Biferale, J. B. Flór, M. Ghil, F. Lott, J. McWilliams, J. Molemaker and J. Sommeria for discussion. Critical remarcs from three anonymous referees have improved the quality of the paper. Calculations were done at IDRIS (France) project: i2012016802.

Edited by: V. I. Vlasenko

Reviewed by: two anonymous referees

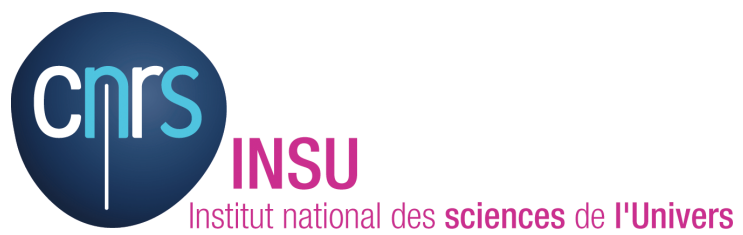

The publication of this article is financed by CNRS-INSU.

\section{References}

Alford, M. H.: Redistribution of energy available for ocean mixing by long-range propagation of internal waves, Nature, 423, 159$162,2003$.

Anderson, D. L. T. and Gill, A. E.: Beta dispersion of inertial Waves, J. Geophys. Res., 84, 1836-1842, 1979.

Bofetta, G. and Ecke, R. E.: Two-Dimensional Turbulence, Ann. Rev. Fluid Mech., 44, 427-451, 2012.

Capet, X., Mc Williams, J. C., Molemaker, M. J., and Shchepetkin, A. F.:, Mesoscale to submesoscale transition in the california current system. Part I: flow structure, eddy flux, and observational tests, J. Phys. Oceanogr., 38, 29-43, 2008.

Chelton, D. B., Schlax, M. G., and Samelson, R. M.: Global Observations of Nonlinear Mesoscale Eddies, Prog. Oceanogr., 91, 167-216, doi:10.1016/j.pocean.2011.01.002, 2011.

Farge, M. and Sadourny, R.: Wave-vortex dynamics in rotating shallow-water, J. Fluid Mech., 206, 433-462, 1990.

Ferrari, R. and Wunsch, C.: Ocean Circulation Kinetic Energy: Reservoirs, Sources, and Sinks, Ann. Rev. Fluid Mech., 41, 253 282, doi:10.1146/annurev.fluid.40.111406.102139, 2009.

Ford, R., McIntyre, M. E., and Norton, W. A.: Balance and the slow quasimanifold: some explicit results, J. Atmos. Scie., 57, 12361254,2000

Ford, R., McIntyre, M. E., and Norton, W. A.: Reply, J. Atmos. Sci., 59, 2878-2882, 2002.

Frisch, U.: Turbulence: the legacy of A.N. Kolmogorov, Cambridge University Press, 1995.

Frisch, U., Kurien, S., Pandit, R., Pauls, W., Ray, S. S., Wirth, A., and Zhu, J.-Z.: Hyperviscosity, Galerkin Truncation, and Bottelnecks in Turbulence, Phys. Rev. Lett., 101, 144501, doi:10.1103/PhysRevLett.101.144501, 2008.

Gent, P. R. and McWilliams, J. C.: Isopycnal Mixing in Ocean Circulation Models, J. Phys. Oceanogr., 20, 150-155, 1990.

Gill, A. E.: Adjustment under gravity in a rotating channel, J. Fluid Mech., 77, 603-621, 1976.

Griffiths, M. and Reeder, M. J.: Stratospheric inertia gravity waves generated in a numerical model of frontogenesis, I: Model solutions, Q. J. Roy. Meteorol. Soc., 122, 1153-1174, doi:10.1002/qj.49712253307, 1996
Klein, P., Hua, B. L., Lapeyre, G., Capet, X., Le Gentil, S., and Sasaki, H.: Upper Ocean Turbulence from HighResolution 3D Simulations, J. Phys. Oceanogr. 38, 1748-1763, doi:10.1175/2007JPO3773.1, 2008.

Kraichnan, R. H.: Inertial ranges in two-dimensional turbulence, Phys. Fluids, 10, 1417-1423, 1967.

Kunze, E.: Near-inertial propagation in geostrophic shear, J. Phys. Oceanogr., 15, 544-565, 1985.

Lighthill, M. J.: On sound generated aerodynamically, I. General Theory, Proc. R. Soc. Lond. A, 211, 564-587, 1952

Lott, F., Plougonven, R., and Vanneste, J.: Gravity waves generated by sheared potential- vorticity anomalies, J. Atmos. Sci., 67 , 157-170, doi:10.1175/2009JAS3134.1, 2010.

McIntyre, M. E.: Spontaneous imbalance and HybridGravity Structures, J. Atmos. Sci., 66, 1315-1326, doi:10.1175/2008JAS2538.1, 2009.

Molemaker, M. J., McWilliams, J. C., and Yavneh, I.: Baroclinic Instability and Loss of Balance, J. Phys. Oceanogr., 35, 15051517, 2005.

O'Sullivan, D. and Dunkerton, T. J.: Generation of inertia-gravity waves in a simulated life cycle of baroclinic instability, J. Atmos. Sci., 52, 3695-3716, 1995

Pedlosky, J.: Waves in the Ocean and Atmosphere, Springer-Verlag, ISBN 3-540-00340-1, 2003.

Plougonven, R. and Snyder, C.: Gravity waves generated by jets: Propagation versus generation, Geophys. Resea. Lett., 30, 1954, doi:10.1029/2003GL017716, 2005.

Polvani, L. M., McWilliams, J. C., Spall, M. A., and Ford, R.: The coherent structures of shallow-water turbulence: Deformationradius effects, cyclone/anticyclone asymmetry and gravity-wave generation, Chaos, 4, 177-186, doi:10.1063/1.166002, 1994.

Reeder, M. J. and Griffiths, M.: Stratospheric inertia gravity waves generated in a numerical model of frontogenesis. II: Wave sources, generation mechanisms and momentum fluxes, Q. J. Roy. Meteorol. Soc., 122, 1175-1195, doi:10.1002/qj.49712253308, 1996.

Rhines, P. B.: Waves and turbulence on a $\beta$-plane, J. Fluid Mech., 69, 417-443, 1975.

Rhines, P. B.: Geostrophic turbulence, Ann. Rev. Fluid Mech., 11, 401-441, 1979

Rossby, C.-G.: On the mutual adjustment of pressure and velocity distributions in certain simple current systems, J. Mar. Res., 1, 239-263, 1938.

Snyder, C., Muraki, D. J., Plougonven, R., and Zhang, F.: InertiaGravity Waves Generated within a Dipole Vortex, J. Atmos. Sci., 64, 4417-4431, doi:10.1175/2007JAS2351.1, 2007.

Vallis, G.: Atmospheric and Oceanic Fluid Dynamics, Cambridge Univ. Press. ISBN: 0-521-84969-1, 2006.

Vanneste, J.: Balance and spontaneous wave generation in geophysical flows, Ann. Rev. Fluid Mech., 45, 147-172, doi:10.1146/annurev-fluid-011212-140730, 2013.

Vanneste, J. and Yavneh, I.: Exponentially small inertia-gravity waves and the breakdown of quasi-geostrophic balance, J. Atmos. Sci., 61, 211-223, 2004.

Zhai, X., Greatbatch, R. J., and Sheng, J.: Doppler-shift inertial oscillations on a $\beta$ plane, J. Phys. Oceanogr., 35, 1480-1488, 2005. 